Relations industrielles

Industrial Relations

\title{
Les relations du travail au Québec : bilan des années 1970 Québec Labour Relations in the 1970's
}

\section{Gérard Hébert}

Volume 36, numéro 4, 1981

URI : https://id.erudit.org/iderudit/029202ar

DOI : https://doi.org/10.7202/029202ar

Aller au sommaire du numéro

Éditeur(s)

Département des relations industrielles de l'Université Laval

ISSN

0034-379X (imprimé)

1703-8138 (numérique)

Découvrir la revue

Citer cet article

Hébert, G. (1981). Les relations du travail au Québec : bilan des années 1970. Relations industrielles / Industrial Relations, 36(4), 715-747.

https://doi.org/10.7202/029202ar
Résumé de l'article

Les règles de base de la négociation collective n'ont guère changé au cours des années 1970. Mais plusieurs de ses paramètres ont considérablement évolué. Le mouvement syndical s'est morcelé, et radicalisé, en partie. Les nouvelles mesures législatives $n$ 'ont pas produit les effets désirés. L'inflation, avec ou sans contrôles, a bouleversé les habitudes de négocier. Le secteur public a pris le pas sur le secteur privé. Le caractère politique de ces rondes majeures risque d'ébranler le régime même de la libre négociation collective, au moins dans ce secteur. Les conventions collectives se sont allongées, et l'approche légaliste s'est accentuée
Tous droits réservés (C Département des relations industrielles de l'Université Laval, 1981
Ce document est protégé par la loi sur le droit d'auteur. L'utilisation des services d'Érudit (y compris la reproduction) est assujettie à sa politique d'utilisation que vous pouvez consulter en ligne.

https://apropos.erudit.org/fr/usagers/politique-dutilisation/ 


\section{Les relations du travail au Québec Bilan des années 1970}

\section{Gérard Hébert}

Les règles de base de la négociation collective n'ont guère changé au cours des années 1970. Mais plusieurs de ses paramètres ont considérablement évolué. Le mouvement syndical s'est morcelé, et radicalisé, en partie. Les nouvelles mesures législatives $n$ 'ont pas produit les effets désirés. L'inflation, avec ou sans contrôles, a bouleversé les habitudes de négocier. Le secteur public a pris le pas sur le secteur privé. Le caractère politique de ces rondes majeures risque d'ébranler le régime même de la libre négociation collective, au moins dans ce secteur. Les conventions collectives se sont allongées, et l'approche légaliste s'est accentuée.

En relations du travail, les années 1970 présentent un caractère complexe et de multiples contradictions. On ne peut en tracer le bilan sans y introduire de sérieuses nuances. Ces années peuvent apparaître, sous un certain angle, comme un grand pas en avant; sous un autre, elles témoignent d'un recul irréparable; elles ont assuré la consolidation de la négociation collective, en même temps qu'elles en ont peut-être préparé la disparition éventuelle. Tel sera le thème de la première partie de cet article. La seconde analysera le contenu des conventions collectives et leur évolution au cours de la décennie. Montréal.

- HÉBERT, Gérard, professeur, École de relations industrielles, Université de

** La première partie de ce texte a été présentée le 6 novembre 1980, au titre de la conférence Jean-Réal Cardin, lors du onzième colloque de relations industrielles de l'Université de Montréal. Elle a déjà paru, sous une forme légèrement différente, dans La négociation collective en question, Onzième colloque de relations industrielles, 1980, Université de Montréal, École de relations industrielles, pp. 9-20. La substance de la seconde partie a fait l'objet d'un exposé plus élaboré, non publié, lors du Congrès scientifique «81», organisé par la Corporation des conseillers en relations industrielles, à Montréal, le 8 mai 1981. 


\section{LA NÉGOCIATION COLLECTIVE}

Rappelons d'abord quelques données de base. Nous évoquerons ensuite les grands événements de la décennie. Nous aborderons alors l'analyse proprement dite.

\section{Les données de base}

Les effectifs syndicaux au Québec sont passés, de 1970 à 1980, d'environ 700,000 à 900,000 , ce qui représente toujours à peu près $37.5 \%$ de la main-d'oeuvre salariée non agricole ${ }^{1}$. Le phénomène peut-être le plus important de la décennie, pour le mouvement syndical québécois, c'est la croissance des syndicats indépendants, plus précisément la désaffiliation de quelques grandes unités, qui a fait passer les effectifs des syndicats non affiliés de $8 \%$ de tous les effectifs syndicaux, au cours des années 1960, à environ 16 ou $17 \%$ à la fin des années $1970^{2}$.

Le nombre des accréditations accordées par le Bureau du commissaire enquêteur était d'environ 1,200 par année, il y a dix ans; il varie aujourd'hui de 800 à $1,000^{3}$. De 3,000 à 4,000 conventions collectives sont déposées chaque année au bureau du Commissaire général du travail ${ }^{4}$. Leur durée moyenne est de deux ans; environ un tiers sont signées pour trois ans.

L'ordinateur du ministère nous disait qu'il y avait en 1978 10,000 conventions collectives en vigueur au Québec dont plus de la moitié régissait des unités de moins de 50 salariés 5 . Une publication de 1979 parle de 8,000 conventions $^{6}$. De ce nombre global, près de 3,000 visent le secteur public et parapublic; il s'agit évidemment de 3,000 unités d'accréditation, dont chacune dépose sa convention collective; on sait que le nombre de conventions collectives distinctes dans le secteur public et parapublic ne dépasse pas 50 . L'information sur le nombre de conventions collectives en vigueur en 1970 n'est pas disponible.

1 Travail Canada, Répertoire des organisations de travailleurs au Canada. Publication annuelle. François DELORME et Gaspar LASSONDE, Aspects de la réalité syndicale québécoise 1976. Études et Recherches $\mathrm{n}^{\circ} 1$, Québec, ministère du Travail et de la Main-d'oeuvre, Direction générale de la recherche, juillet 1978, $42 \mathrm{p}$.

2 DELORME, François, Les syndicats indépendants au Québec: un aperçu de leur situation, Québec, ministère du Travail et de la Main-d'oeuvre, Centre de recherche et de statistiques sur le marché du travail, juin 1980.

3 Québec, ministère du Travail et de la Main-d'oeuvre, Rapport annuel.

4 Ibid.

5 Québec, ministère du Travail et de la Main-d'oeuvre, Direction générale de la recherche, données mécanographiques non publiées, août 1978.

6 "Analyse de statistiques sur les conventions collectives», Centre de recherche et de statistiques sur le marché du travail, Québec, ministère du Travail et de la Main-d'oeuvre, Direction des communications, s.d. (1979?), p. 21. 
On ignore la durée moyenne des négociations au Québec. Mais on sait que les grandes négociations au Canada (celles qui visent 500 travailleurs et plus) durent en moyenne six mois; $10 \%$ prennent plus d'une année. La situation n'a pas changé depuis dix ans'. Quant à l'étape où le règlement s'effectue, $40 \%$ des grandes conventions canadiennes se règlent à l'étape de la négociation directe, $45 \%$ grâce à l'intervention d'un tiers et $15 \%$ suite à un arrêt de travail. Cette proportion non plus n'a guère changé depuis dix ans.

Pour ce qui regarde le contexte, l'inflation constitue le phénomène majeur de la décennie. Au cours des premières années, l'indice des prix à la consommation augmentait de 2 à 3\% par année 8 . En 1974 et 1975, nous avons eu près de $11 \%$ d'augmentation annuelle des prix; tombée à $7.5 \%$ en 1976 , l'inflation dépasse de nouveau $10 \%$ en 1980 . En somme, les prix ont doublé depuis dix ans. En réponse à la poussée des prix au milieu de la décennie, la proportion des grandes conventions canadiennes incluant une clause d'indexation a augmenté considérablement, passant d'environ $20 \%$ au début de la décennie à $45 \%$ à la fin de 1976 , pour retomber à $35 \%$ l'année dernière9.

Pour ce qui est du taux de chômage, il a oscillé entre 5 et $8 \%$ dans l'ensemble du Canada, entre 7 et $11 \%$ au Québec. Il s'établit maintenant à 7 et $10 \%$ respectivement ${ }^{10}$.

Quant aux gains horaires moyens dans l'industrie manufacturière, ils sont passés de $\$ 3$ à $\$ 8$ au Canada, et à $\$ 7.50$ au Québec. Les gains hebdomadaires moyens étaient $\$ 135$ en 1970; ils sont maintenant $\$ 315^{11}$. En comparant ainsi les principales données de 1970 à celles de 1980, on peut conclure que les gains moyens ont augmenté plus vite que les prix; si on choisissait comme base une autre donnée, on pourrait prouver le contraire.

\section{Les grands événements}

Si nous jetons maintenant un coup d'oeil sur les principaux événements de la décennie, on peut noter ce qui suit.

C'est d'emblée le secteur public, avec ses trois rondes de négociation, qui a dominé la scène de la négociation collective. Chaque ronde ayant ame-

7 Travail Canada, Revue de la négociation collective, 1971-1980.

8 L'indice des prix à la consommation, Statistique Canada 62-001, 1970-1980.

9 Travail Canada, "Clauses d'indemnité de vie chère dans les grandes conventions collectives», Données sur le travail, Travail Canada, décembre 1979, 5 et $20 \mathrm{p}$.

10 La population active, Statistique Canada 71-001, 1970-1980.

11 Emploi, gains et durée du travail, Statistique Canada 72-002, 1970-1980. 
né une série de grèves, concentrées ou successives, le nombre de jourshommes perdus par suite d'arrêts de travail au Québec a dépassé les 3,000,000 en 1972, en 1975 et 1976, en 1979 et 1980. En 1976, une grève de la construction et des manifestations contre les mesures anti-inflation ont porté le total à plus de $6,000,000$ de jours perdus ${ }^{12}$. Par rapport à l'ensemble des jours travaillés au Québec, la proportion atteint un niveau qui brise tous les records, $1.15 \%$ en $1976,0.6 \%$ en 1972,1975 et 1979 . La proportion pour 1980 sera vraisemblablement dans le même ordre de grandeur.

L'application des mesures anti-inflationnistes semble bien avoir été l'événement majeur pour le secteur privé de l'économie. Proclamées le 14 octobre 1975, elles s'appliquèrent jusqu'à la fin de $1978^{13}$. Nous y reviendrons tantôt.

Faut-il rappeler les grands conflits du secteur privé? La Presse en 1970 et 1971, les grèves de Joliette (Firestone et Canadium Gypsum) en 1973 et 1974, puis successivement, United Aircraft, l'amiante, l'Alcan, Commonwealth Plywood et Kenworth, les mines de la Côte-Nord et de la Gaspésie, l'industrie des pâtes et papiers ${ }^{14}$.

Dans le domaine de la législation, beaucoup de lois visaient le secteur public, soit pour déterminer le cadre de la négociation à venir, soit pour forcer le retour au travail. Pour le secteur privé, à part la construction, qui a eu droit en moyenne à une loi par année, le Code du travail n'a connu qu'une modification importante: introduite sous le numéro 24 en 1974, elle a finalement été adoptée, après modifications et ajouts, le 22 décembre 1977; on y réfère habituellement sous le nom poétique de Projet de loi $n^{\circ} 45^{15}$. Il ne faut pas oublier, évidemment, la Loi anti-inflation, adoptée respectivement, au fédéral et au provincial, le 3 et le 19 décembre $1975^{16}$. Enfin, en 1979, il y

12 DELORME, François, Gaspar LASSONDE, Lucie TREMBLAY, Grèves et lock-out au Québec, 1966-1976. Quelques précisions sur les modes de compilation, Québec, ministère du Travail et de la Main-d'oeuvre, Direction générale de la recherche, octobre 1977, 36 p. Grèves et lock-out au Québec, Rapport annuel, Québec, ministère du Travail et de la Maind'oeuvre, Direction générale de la recherche. Grèves et lock-out au Canada, Annuel ou bisannuel, Travail Canada, 1970-1980.

13 Loi ayant pour objet de limiter les marges bénéficiaires, les prix, les dividendes et les rémunérations au Canada (Loi anti-inflation). (Bill C-73, sanctionné le 3 décembre 1975). Statuts du Canada, 1975, ch. 75.

14 Voir la note 12.

15 Loi modifiant le Code du travail et la Loi du ministère du Travail et de la Maind'oeuvre. (Projet de loi nº 45, sanctionné le 22 décembre 1977). Lois du Québec, 1977, ch. 41.

16 Loi anti-inflation. Statuts du Canada, 1975, ch. 75. Loi concernant les mesures antiinflationnistes. (Projet de loi ${ }^{\circ}$ 64, sanctionné le 19 décembre 1975). Lois du Québec, 1975, ch. 16. 
eut la Loi sur les normes du travail et la Loi sur la santé et la sécurité du travail, mais qui ne touchent qu'indirectement la négociation collective ${ }^{17}$.

La prédominance du secteur public sur le secteur privé, que nous venons de relever dans les conflits et dans la législation, constitue peut-être la caractéristique principale de la négociation collective au cours des derniers dix ans. Avant de nous engager dans une comparaison entre les deux secteurs, il faut d'abord considérer les deux séparément. Voyons d'abord le secteur privé.

\section{Le secteur privé}

Les trois années que se sont appliquées les mesures anti-inflationnistes constituent une sorte de parenthèse dans la négociation collective au Canada pendant la décennie de 1970. Les représentants syndicaux ont proclamé que c'était la mort de la négociation, alors que les employeurs y trouvaient à la fois des avantages et des inconvénients. Quel que soit le jugement que l'on porte sur ces mesures, il reste qu'elles semblent avoir raccourci sinon facilité le processus pendant ces trois années. Les chiffres sont clairs pour 1977 et 1978, où le pourcentage des jours perdus, tant au Québec qu'au Canada, est tombé à .2 ou $.3 \%$; pour ce qui est de la durée des grandes négociations, la proportion des règlements avant 6 mois a augmenté, et celle des cas qui ont pris plus d'un an à se négocier a considérablement diminué18. La situation en 1976 est moins claire: au Québec, c'est l'année des six millions et demi de jours perdus. Il faut se rappeler que plus de la moitié s'explique par des arrêts de travail dans deux secteurs, la construction et le secteur parapublic. Une fois les mesures anti-inflationnistes levées, les indicateurs ont repris leur position habituelle.

L'effet du Projet de loi 45 sera plus durable; mais son influence semble avoir été moindre qu'on ne l'avait escompté. Les principales modifications qu'il apportait à notre régime de négociation collective ne semblent pas avoir changé considérablement la réalité.

Même volontaire, les parties demandent la conciliation presque aussi souvent qu'auparavant, et la coopération qu'elles apportent au conciliateur ne s'est pas accrue de façon spectaculaire ${ }^{19}$. Le modèle général n'a pas changé pour la peine, sauf sur un point évidemment: on ne demande plus la conciliation pour déterminer la date de l'exercice légal du droit de grève. Mais

17 Loi sur les normes du travail. (Projet de loi $n^{\circ} 126$, sanctionné le 22 juin 1979). Lois $d u$ Québec, 1979, ch. 45. Lois sur la santé et sur la sécurité du travail. (Projet de loi n ${ }^{\circ} 17$, sanctionné le 21 décembre 1979). Lois du Québec, 1979, ch. 63.

18 Voir les notes 7 et 12.

19 Québec, ministère du Travail et de la Main-d'oeuvre, Rapport annuel, 1978-1979, p. 8. «La conciliation volontaire a amené la signature de 12 conventions collectives», Le journal du travail, 1, 4, août 1979, p. 4. 
on demande l'intervention du conciliateur comme avant, par habitude sans doute: pour impressionner l'autre partie, pour faire avancer la discussion, ou tout simplement pour avoir un atout de plus dans son jeu.

L'arbitrage de la première convention collective a permis de régler, en 1978 et 1979, une dizaine de cas sur une cinquantaine de demandes d'intervention; il faudrait une analyse plus approfondie pour savoir si la douzaine d'autres cas qui se sont réglés avant la décision arbitrale l'ont été par suite de la disposition coercitive que l'employeur pouvait craindre. En ce cas, il faudrait également attribuer le règlement de ces cas à la disposition ellemême, ce qui porterait à environ $50 \%$ les cas réglés par rapport au nombre de demandes déposées ${ }^{20}$.

L'application automatique de la formule Rand a apporté aux syndicats en formation une aide financière importante: ils n'ont plus à attendre la signature de la première convention collective pour s'assurer une rentrée de fonds régulière. La nature de la mesure en garantit les résultats dès sa mise en application. Des études ultérieures nous diront si la meilleure position financière des jeunes syndicats leur a permis de régler plus rapidement leur première convention, et si elle a diminué le taux d'échecs ou de faux départs.

Les dispositions les plus spectaculaires du Projet de loi 45, les mesures anti-scabs, se sont avérées difficiles d'application ${ }^{21}$. Plusieurs enquêtes ont été demandées et effectuées; plusieurs ont fait l'objet d'appels aux tribunaux $^{22}$. Les mesures ne semblent pas avoir été l'instrument infaillible qui devait assurer l'arrêt de la production: ou l'employeur utilise les possibilités qui lui sont offertes par la loi, ou il décide carrément de passer outre, comme on l'a vu dans certains cas, où les employeurs ont déclaré l'avoir fait au nom d'impératifs humanitaires supérieurs. Les mesures ont-elles réalisé l'objectif principal que l'on disait poursuivre, soit la diminution de la violence? Il est certain que la violence n'a pas cessé totalement; certains prétendent même qu'elle n'a pas diminué du tout, qu'on lui accorde tout simplement moins de publicité dans les médias d'information qu'on ne le faisait il y a cinq ou six ans.

20 GIRARD, Michelle, "L'arbitrage des premières conventions collectives», Le marché du travail, 1, 3, juillet 1980, pp. 40-43.

21 MARTINEAU, Luc, "L'expérience des dispositions anti-briseurs de grève selon le Code du travail (Québec)", Relations industrielles, 35, 3, 1980, pp. 555-569.

22 Métallurgistes Unis d'Amérique Syndicat local 6833 (FTQ) c. Société d'Énergie de la Baie James, (1979) C.S. 738. Syndicat des Placeurs et des Ouvreuses de la Place des Arts (CSN et al c. Régie de la Place des Arts, C.S. 500-05-000212-801, 6 février 1980, juge Ruston B. Lamb, non rapporté. Texaco Canada Limitée c. Bastien et Syndicat international des travailleurs des industries pétrolière, chimique et atomique local 9-70 et le P.G. du Québec (mis-encause), C.A. 500-09-000437-780, 18 janvier 1980, Juges Turgeon, Mayrand et Monet, non rapporté. 
En un mot, pas de changement spectaculaire dans le processus de la négociation pour le secteur privé, sauf peut-être dans un cas, celui de l'industrie de la construction. L'instauration d'une formule de négociation sectorielle et de la syndicalisation obligatoire, à la fin des années 1960, avait promis un âge d'or aux relations de travail dans cette industrie ${ }^{23}$. La réalité s'est avérée bien différente: grèves à chaque ronde de négociation, loi spéciale dans au moins trois conflits ${ }^{24}$, commission d'enquête majeure ${ }^{25}$, qui a amené une intervention gouvernementale encore plus grande, entre autres sous la forme de tutelles ${ }^{26}$, effort d'un groupe syndical particulier pour établir un monopole, qui n'aboutit qu'à une division encore plus considérable ${ }^{27}$. Sous plusieurs aspects les relations du travail dans l'industrie de la construction ressemblent à celles du secteur public: législation fréquente, réglementation abondante, fin de la négociation par décision gouvernementale aussi souvent que par l'accord des parties ${ }^{28}$. La négociation sectorielle semble appeler une intervention gouvernementale massive, presque autant que la négociation du secteur public et parapublic. C'est maintenant vers ce secteur public qu'il faut tourner notre attention.

23 Loi sur les relations de travail dans l'industrie de la construction. (Projet de loi $\mathrm{n}^{\circ} 290$, sanctionné le 18 décembre 1968). Statuts du Québec, 1968, ch. 45.

24 Loi concernant l'industrie de la construction. (Projet de loi n ${ }^{\circ} 38$, sanctionné le 8 août 1970). Lois du Québec, 1970, ch. 34. Loi modifiant de nouveau la Loi sur les relations du travail dans l'industrie de la construction. (Projet de loi ${ }^{\circ} 14$, sanctionné le 22 décembre 1973). Lois du Québec, 1973, ch. 29. Loi modifiant la Loi sur les relations du travail dans l'industrie de la construction. (Projet de loi ${ }^{\circ}$ 201, sanctionné le 24 décembre 1974), Lois du Québec, 1974 , ch. 38. On pourrait aussi nommer la Loi modifiant la Loi sur les relations du travail dans l'industrie de la construction. (Projet de loi $\mathrm{n}^{\circ}$ 9, sanctionné le $1^{\mathrm{er}}$ juin 1973), Statuts $d u$ Québec, 1973, ch. 28, Loi modifiant de nouveau la Loi des syndicats professionnels et d'autres dispositions législatives. (Projet de loi $\mathrm{n}^{\circ}$ 58, sanctionné le 8 juillet 1972), Lois du Québec, 1972, ch. 63.

25 Rapport de la Commission d'enquête sur l'exercice de la liberté syndicale dans l'industrie de la construction, Juge Robert Cliche, président, Québec, Éditeur officiel du Québec, 1975, $355 \mathrm{p}$.

26 Loi sur la mise en tutelle de certains syndicats ouvriers. (Projet de loi $\mathrm{n}^{\circ} 29$, sanctionné le 22 mai 1975), Lois du Québec, 1975, ch. 57. Voir aussi Loi sur la mise en tutelle de "International Union Elevator Constructors, Locals 89 and 101". (Projet de loi ${ }^{\circ} 43$, sanctionné le 17 juillet 1974), Lois du Québec, 1974, ch. 116.

27 Loi modifiant la Loi sur les relations du travail dans l'industrie de la construction et concernant la représentativité de certaines associations représentatives. (Projet de loi $\mathrm{n}^{\circ} 109$, sanctionné le 4 décembre 1980), Lois du Québec, 1980, ch. 23.

28 HÉBERT, Gérard, Les relations du travail dans la construction au Québec, Ottawa, Conseil économique du Canada, 1977, 206 p. et 1978, 273 p. Gérard HÉBERT, «La négociation sectorielle: le régime des décrets dans l'industrie de la construction», dans La gestion des relations du travail au Québec, publié sous la direction de Noël Mallette, Montréal, McGrawHill, c. 1980, pp. 513-529. 


\section{Le secteur public}

Tout comme pour la construction, les règles fondamentales de la négociation dans les secteurs public et parapublic avaient été posées au cours des années $1960^{29}$. Elles n'ont cependant pris leur forme arrêtée et leur consistance qu'au cours de la dernière décennie. Même si les trois rondes de négociation de la décennie présentent des ressemblances fondamentales entre elles, elles comportent des différences importantes.

La ronde de 1972 apparaît comme la plus centralisée et la plus percutante des trois ${ }^{30}$. L'alliance sacrée entre les trois centrales et les différents secteurs a toujours été un peu branlante. Les objectifs principaux de chacun des trois groupes majeurs étaient trop différents: salaire de base pour les employés d'hôpitaux, sécurité d'emploi pour les enseignants, questions d'évaluation pour les fonctionnaires. Pourtant le front commun s'est maintenu, depuis la prénégociation, au début de 1971, jusqu'aux grands affrontements d'avril et mai 1972. On se souvient de ceux-ci: grève illimitée de presque tout le secteur public et parapublic depuis le 11 avril, défi de la loi spéciale adoptée le 21 avril ${ }^{31}$, emprisonnement des trois présidents de centrales le 9 mai, grèves sporadiques à travers la province du 9 au 12 mai. Tout de suite après ces événements, l'effritement du front commun s'est affirmé de façon définitive: les syndicats des employés d'Hydro-Québec s'en étaient retirés le 13 avril; un groupe de dissidents de la C.S.N., expulsés le 28 mai, fondent la C.S.D. le 8 juin; le S.F.P.Q. se désaffilie de la C.S.N. au cours de l'été32. La ronde se termina par des ententes successives: les employés d'hôpitaux signèrent le 16 octobre, ceux de la S.A.Q. le 27 octobre; quant aux fonctionnaires, qui avaient quitté le front commun le 14 août, ils signè-

29 Code du travail. (Projet de loi ${ }^{\circ} 54$, sanctionné le 31 juillet 1964), Statuts du Québec, 1964, ch. 45, art. 99. Loi sur la fonction publique. (Projet de loi $\mathrm{n}^{\circ} 55$, sanctionné le 6 août 1965), Statuts du Québec, 1965, ch. 69. Loi assurant le droit de l'enfant à l'éducation et instituant un nouveau régime de convention collective dans le secteur scolaire. (Projet de loi $n^{\circ} 25$, sanctionné le 17 février 1967), Statuts du Québec, 1966-67, ch. 63.

30 BOIVIN, Jean, The Evolution of Bargaining Power in the Province of Quebec Public Sector (1964-1972), Collection: Relations du travail, Québec, Université Laval, Département des relations industrielles, janvier 1975, 359 p. Jean BOIVIN, «La négociation collective dans le secteur public québécois, une évaluation des trois premières rondes (1964-1972)», Relations industrielles, 27, 4, octobre 1972, pp. 679-708. Gérard HÉBERT, «La négociation du secteur public provincial: histoire et cadre institutionnel», dans La gestion des relations du travail au Québec, publié sous la direction de Noël Mallette, Montréal, McGraw-Hill, c. 1980, pp. 547-569.

31 Loi assurant la reprise des services dans le secteur public. (Projet de loi $\mathrm{n}^{\circ}$ 19, sanctionné le 21 avril 1972), Lois du Québec, 1972, ch. 7.

32 HÉBERT, Gérard, «Les relations du travail dans la fonction publique au Québec: situation et orientations», Relations industrielles, 29, 4, décembre 1974, pp. 750-772. 
rent le 24 octobre; pour les enseignants, le gouvernement adopta, en décembre, le décret qui devait leur servir de convention collective jusqu'à la prochaine ronde ${ }^{33}$.

Au cours de la ronde de 1975-1976, le front commun n'eut jamais la cohérence de la ronde précédente: le S.F.P.Q. mena sa négociation séparément et signa sa convention collective en décembre 1975, six mois avant la fin des négociations dans le secteur des affaires sociales et un an avant celle du secteur de l'enseignement. Cette ronde nous a donné le commissaire aux services essentiels et les commissaires aux différends scolaires ${ }^{34}$; dans le cas des hôpitaux, il a fallu en plus une loi spéciale de retour au travail ${ }^{35}$. On connaît la ronde de 1979-1980, qui comporte également sa loi spéciale, en novembre $1979^{36}$, et dont les négociations complémentaires, pour les commissions scolaires, ne sont pas encore terminées, deux d'entre elles venant tout juste de faire l'objet d'une autre loi spéciale ${ }^{37}$. Depuis 1970 , le gouvernement a ainsi adopté 12 lois spéciales pour mettre fin à autant de conflits, dont 10 touchaient le secteur public ${ }^{38}$.

Les trois rondes ont démontré de plus en plus clairement le caractère essentiellement politique de l'exercice, et l'équivoque qu'il prolonge du fait qu'il se présente sous l'étiquette d'une négociation collective. Les objectifs,

33 Québec, Décret tenant lieu de convention collective entre les instituteurs et les commissions scolaires et les commissions régionales, Arrêté en conseil $\mathrm{n}^{\circ}$ 3811-72 du 15 décembre 1972, $132 \mathrm{p}$.

34 Loi visant à assurer les services de santé et les services sociaux essentiels en cas de conflit de travail. (Projet de loi $\mathrm{n}^{\circ}$ 253, sanctionné le 19 décembre 1975), Lois du Québec, 1975, ch. 52. Loi concernant le maintien des services dans le domaine de l'éducation et abrogeant une disposition législative. (Projet de loi n ${ }^{\circ} 23$, sanctionné le 9 avril 1976), Lois du Québec, 1976, ch. 38.

35 Loi concernant les services de santé dans certains établissements. (Projet de loi $\mathrm{n}^{\circ} 61$, sanctionné le 24 juillet 1976), Lois du Québec, 1976, ch. 29.

36 Loi sur les propositions aux salariés des secteurs de l'éducation, des affaires sociales et de la fonction publique. (Projet de loi $\mathrm{n}^{\circ}$ 62, sanctionné le 12 novembre 1979), Lois du Québec, 1979 , ch. 50.

37 Loi sur certains différends entre des enseignants et des commissions scolaires. (Projet de loi $n^{\circ} 113$, sanctionné le 24 octobre 1980), Lois du Québec, 1980, ch. 22.

38 Voir les notes $31,34,35,36$ et 37 . Il y eut également les lois spéciales suivantes. Loi sur les services essentiels d'Hydro-Québec. (Projet de loi n 73, sanctionné le 15 novembre 1972), Lois du Québec, 1972, ch. 9. Loi assurant aux usagers la reprise des services normaux de la Commission de transport de la Communauté urbaine de Montréal. (Projet de loi ${ }^{\circ} 57$, sanctionné le 27 septembre 1975), Lois du Québec, 1975, ch. 56. Loi assurant le maintien des services d'électricité et prévoyant les conditions de travail des salariés d'Hydro-Québec. (Projet de loi $n^{\circ} 88$, sanctionné le 18 décembre 1979), Lois du Québec, 1979, ch. 62. Loi assurant la reprise de certains services de la ville de Montréal et de la Communauté urbaine de Montréal. (Projet de loi n ${ }^{\circ} 93$, sanctionné le 24 mars 1980), Lois du Québec, 1980, ch. 1. Dans le domaine de la construction, il y eut les lois mentionnées dans la note 24 , ainsi que la Loi modifiant la Loi sur les relations de travail dans l'industrie de la construction. (Projet de loi ${ }^{\circ}{ }^{15}$, sanctionné le 24 mars 1972), Lois du Québec, 1972, ch. 10. 
même strictement économiques, sont formulés sous couleur de mesures sociales. Le salaire de base réclamé dans le secteur des affaires sociales, au cours des trois rondes, $\$ 100, \$ 165$ et $\$ 265$, a toujours été justifié parce qu'il représentait le minimum d'un salaire décent et familial, que l'État se devait de donner à ses employés, pour entraîner à sa suite le secteur privé. L'impact socio-politique du congé de maternité paraît tout aussi clair.

Quant au processus lui-même, il est également de nature politique. Les divers arrangements qu'on a voulu mettre en place au cours de chacune des rondes de négociation, du côté patronal, pour équilibrer en quelque sorte la représentation gouvernementale et celle des employeurs plus immédiats, les commissions scolaires et les hôpitaux, n'ont guère affecté le déroulement du processus ${ }^{39}$. Les représentants syndicaux savent bien que, pour obtenir le maximum, ils doivent s'adresser directement à l'État, au pouvoir suprême: les négociations avec les représentants patronaux ne servent ainsi qu'à préparer les pressions dernières sur le pouvoir politique, qui seul possède en mains toutes les cartes du jeu. Sans lui accorder plus d'importance qu'il n'avait, le slogan de 1972 «Il faut casser le régime» n'est-il pas révélateur du caractère politique d'un affrontement de cette nature et de cette envergure? ? $^{40}$

Le résultat n'est peut-être pas aussi clairement politique que le processus lui-même. En tout cas, ses conséquences économiques sont importantes y compris pour le secteur privé. Pour n'en nommer que trois, le niveau d'augmentation des salaires, la durée des vacances payées et le congé de maternité ont exercé et continuent d'exercer un effet d'entraînement dans le secteur privé. L'effet secondaire sur le niveau de chômage est plus difficile à déterminer. Une chose semble certaine, c'est que la différence des conditions de travail entre le secteur privé et le secteur public s'agrandit de ronde en ronde de négociation.

39 Loi du régime de négociations collectives dans les secteurs de l'éducation et des hôpitaux. (Projet de loi $\mathrm{n}^{\circ} 46$, sanctionné le 30 juin 1971), Lois du Québec, 1971, ch. 12. Loi sur les négociations collectives dans les secteurs de l'éducation, des affaires sociales et des organismes gouvernementaux. (Projet de loi $\mathrm{n}^{\circ}$ 95, sanctionné le 24 décembre 1974), Lois du Québec, 1974, ch. 8. Loi sur l'organisation des parties patronale et syndicale aux fins des négociations collectives dans les secteurs de l'éducation, des affaires sociales et des organismes gouvernementaux. (Projet de loi no 55, sanctionné le 23 juin 1978), Lois du Québec, 1978, ch. 14.

40 Voir par exemple la déclaration du président de la C.E.Q. rapportée dans Le Soleil, 5 juin 1972, p. 3. Voir aussi La politisation des relations du travail, compte rendu des travaux du vingt-huitième congrès des relations industrielles de l'Université Laval, Québec, Presses de l'Université Laval, 1973, 169 p., surtout les articles de Jean BOIVIN: «L'impact des secteurs public et parapublic sur la politisation des relations du travail», pp. 66-71 et Gérard DION: «Les conséquences de la politisation des relations du travail», pp. 148-149. 
Alors que le secteur public de caractère provincial est syndiqué à près de $100 \%$, le secteur privé ne l'est qu'à environ 25 ou $30 \%$, et encore, inégalement selon le secteur, secondaire, primaire ou tertiaire ${ }^{41}$. Et si les 25 ou $30 \%$ du secteur privé réussissent tant bien que mal à suivre la locomotive du secteur public, les 70 à $75 \%$ de non syndiqués du secteur privé demeurent beaucoup plus loin en arrière. Une différence majeure entre les deux secteurs, c'est que les employés du secteur public jouissent d'une sécurité considérable, quasi totale ${ }^{42}$, alors que ceux du secteur privé en ont à peine, en tout cas, pas plus que les entreprises elles-mêmes. Par rapport aux griefs, le secteur public est également avantagé: la définition du grief y est généralement très étendue, et l'employeur, c'est-à-dire l'État, a consenti à payer la totalité des frais dans bien des cas, alors que dans le secteur privé, la définition est souvent restreinte, et les frais doivent être partagés moitié moitié43. Quant aux conditions salariales, si la rémunération du secteur public retardait, pour plusieurs classifications, au début des années 1970, on s'accorde généralement pour dire qu'en 1980, elle est au moins égale sinon meilleure pour presque toutes les catégories, sans compter les nombreux avantages additionnels qu'on retrouve dans le secteur public.

\section{Évolution et caractéristiques}

Sur cet arrière-plan de l'importance du secteur public et de ses répercussions sur le secteur privé, on peut dégager les caractéristiques suivantes de la décennie passée.

Tout en gardant fondamentalement une attitude d'agent négociateur, le syndicalisme s'est coloré davantage d'une idéologie de gauche, plus ou moins poussée selon les cas. L'observation est plus vraie de deux centrales qu'on trouve massivement dans le secteur public 44 ; mais de nouveau, il y a eu un effet d'entraînement sur certains secteurs ou services de l'autre centrale ${ }^{45}$. Ainsi, le mouvement syndical oscille entre des positions idéologiques

41 DELORME, François et Gaspar LASSONDE, Aspects de la réalité syndicale québécoise 1976. Québec, ministère du Travail et de la Main-d'oeuvre, juillet 1978, pp. 7-11. François DELORME, "Quelques données sur la syndicalisation au Québec en 1977», Le marché du travail, vol. 1, no 1, mai 1980, p. 35.

42 Voir, par exemple, la Convention collective intervenue entre le comité patronal de négociation du secteur des Affaires sociales et la Fédération des affaires sociales (C.S.N.), 27 mars 1980-31 décembre 1982, art. 15.03.

43 Ibid., art. 10.00 et 11.35 .

44 Voir par exemple, C.S.N.: Ne comptons que sur nos propres moyens, octobre 1971. C.E.Q.: L'école au service de la classe dominante, juin 1972. Voir aussi 150 ans de luttes. Histoire du mouvement ouvrier au Québec (1825-1976), Montréal, co-édition C.S.N.-C.E.Q., $1979,235 \mathrm{p}$.

45 F.T.Q., L'État, rouage de notre exploitation, décembre 1971. 
avancées et sa fonction de caractère économique. Ceci entraîne la radicalisation de plusieurs demandes syndicales et de plusieurs conflits. C'est peutêtre là aussi une raison, parmi d'autres, qui explique qu'on passe souvent outre aux prescriptions légales, y compris aux injonctions et même parfois aux lois spéciales.

Cette radicalisation, bien qu'on la retrouve dans le secteur privé, paraît plus importante dans le secteur public. La centralisation des structures, de part et d'autre, éloigne la base des niveaux de décision et augmente les frustrations: le sentiment d'impuissance invite à se tourner vers des solutions radicales. Le rôle accru des permanents facilite aussi l'infiltration de certains militants révolutionnaires. La radicalisation d'un côté entraîne un durcissement de l'autre. Que ce soit par un mouvement de réaction, par tradition ou par nature, la partie patronale a eu tendance à manifester plus d'intransigeance. On peut en voir un indice dans l'augmentation des recours au lock-out, même dans le secteur public ${ }^{46}$.

Le processus lui-même, j'allais dire le rituel de la négociation, n'a guère changé au cours de la décennie. Malgré un léger raccourcissement en 1977 et 1978, les négociations demeurent aussi longues en 1980 qu'elles l'étaient en 1970. La proportion de celles qui durent de 10 à 12 mois, ou plus de 12 mois, a même augmenté en 197947. Le recours au bluff et au jeu de la résistance demeure presque dans tous les cas un élément inéluctable et nécessaire du jeu de la négociation. Bien fou serait celui qui, pour hâter un processus devenu démesurément long, tenterait de faire lui-même les premières concessions avant que le temps ne soit mûr pour le règlement. Il aurait tôt fait de se faire remettre à l'ordre pas ses mandants, qu'il s'agisse d'une assemblée syndicale ou de la haute direction d'une entreprise. Il faut donner l'impression qu'on s'est battu jusqu'au dernier souffle. La réflexion que l'on faisait il y a dix ans vaut encore aujourd'hui: «On aurait pourtant pu arriver à la même entente en moins de temps et à bien moins de frais.» Tous sont d'accord, mais personne n'est prêt à bouger pour changer quelque chose. Et ce n'est pas facile: il faudrait modifier les attentes et les opinions des syndiqués, tout autant que celles des grands administrateurs.

Tout semble entretenir les parties dans leurs attitudes de contestation et d'affrontement. La lenteur du processus y contribue, mais aussi la nécessité de maintenir le moral des troupes, de part et d'autre: les prises de position répétées rendent plus difficiles ensuite les inévitables retraits stratégiques.

46 Le 23 juin 1978, une modification au Code du travail prévoyait explicitement la possibilité d'un lock-out dans les secteurs public et parapublic. Loi modifiant le Code du travail (Projet de loi $\mathrm{n}^{\circ}$ 59, sanctionné le 23 juin 1978), Lois du Québec, 1978, ch. 52, art. 4, ajoutant divers articles au Code, dont $99 k$ et $99 l$ (aujourd'hui art. 111.11 et 111.12).

47 Travail Canada, Revue de la négociation collective, 1977-1980. 
Les gains réalisés attisent les appétits, et les grands profits déclarés par certaines compagnies laissent croire que tout est possible partout. Tout cela maintient un climat d'affrontement. Pourtant, l'entente suppose et exige un minimum de coopération et d'harmonie. La collaboration patronaleouvrière a aussi mauvaise presse en 1980 qu'en 1970; mais comme elle est aussi nécessaire qu'à ce moment, on lui trouve de nouveaux noms, médiation préventive, négociation permanente ${ }^{48}$.

Les arrêts de travail manifestent certains caractères nouveaux. Il y a plus de grèves illégales. Il y a dix ans, on réclamait le droit de grève en tout temps; aujourd'hui, on le prend, si on pense s'en tirer avec une absolution générale dans le protocole de retour au travail. De façon plus générale, légales ou illégales, les grèves sont plus dispersées et multiformes: grèveavertissement, grève-surprise, grève rotative, grève d'appui. Révélatrice de cette transformation est la comparaison entre la grève du front commun, en 1972, qui a tout bloqué du 11 au 22 avril, et celles de la dernière ronde, qui se sont échelonnées tout au long de 1979 et de 1980 . On semble aussi moins se préoccuper de la loi, des injonctions et même de l'opinion publique. Inversement, bien que ce soit moins fréquent, on commence à trouver des syndiqués qui décident carrément de franchir des piquets de grève s'ils sont en désaccord avec leurs confrères. Le piquet de grève commence à perdre de son caractère quasi religieux qu'on lui avait longtemps attribué. Qu'il s'agisse de passer outre à la loi ou à un piquet de grève, chacun essaie d'évaluer les conséquences de son geste. Il faut ici mentionner le sentiment de crainte que plusieurs expriment en sourdine, mais ressentent vivement semble-t-il. Il est étonnant que l'on entende si souvent évoquer, en conversation privée évidemment, l'argument de la crainte dans un processus qui devrait être, à cause de sa nature même et de son importance, essentiellement démocratique.

Si la lenteur et les méandres du processus de négociation causent des problèmes à tant de personnes - direction, salariés, public - quelques autres personnes en tirent profit. Plus la négociation se prolonge, plus les avocats et les conseillers en relations industrielles qui y participent, à titre de consultants, reçoivent d'honoraires. Un avantage semblable leur résulte aussi de la longueur de plus en plus considérable des conventions collectives. Celles-ci ont continué, au cours de la dernière décennie, à s'allonger, à se nuancer, à devenir plus subtiles et plus exhaustives, à tel point que seuls les spécialistes peuvent s'y retrouver. Pourtant l'objectif de ces documents était d'assurer aux travailleurs de bonnes conditions de travail, ainsi qu'une jus-

48 "Création d'un service de médiation préventive», Le Journal du travail, 2, 2, avril 1980, p. 1. "A Solution: A New Social Contract. Creating a New Sense of Teamwork", Business Week, June 30, 1980 (Special issue on “The Reindustrialization of America”), pp. 86-87. 
tice rapide et d'accès facile pour eux. Mais il est dans la logique du système d'avoir des textes qui prévoient à peu près tous les cas possibles et qui couvrent de plus en plus de matière.

Un phénomène qui ne modifiera probablement pas cette tendance, mais qui l'amènera peut-être à s'orienter autrement, c'est l'adoption de lois et de règlements, et la création d'organismes qui rendent des décisions dans des domaines touchant à la négociation collective. Je parle ici de lois comme la Loi sur les normes du travail, la Loi sur la santé et la sécurité du travail, la Charte des droits de la personne, la Charte de la langue française, la Loi sur la qualité de l'environnement ${ }^{49}$; toutes ces lois prévoient des règlements qui touchent à des points faisant déjà l'objet ou qui auraient pu faire l'objet de dispositions dans les conventions collectives. Ces lois multiplient également les recours, à tel point que pour un même événement le salarié peut s'adresser à trois ou quatre organismes différents, de façon parallèle ou successive $^{50}$. La multiplication de règlements et d'organismes de cette nature vise à favoriser le travailleur; mais est-ce vraiment assuré? il se peut que ces ouvertures engagent le travailleur dans des procédures si complexes et si coûteuses que le résultat, au bout du compte, quel qu'il soit, compense à peine pour les efforts qu'il y aura mis, et pour les inquiétudes qu'il aura subies. De toute façon, la société doit en payer le prix.

Quant aux résultats de la négociation dans le secteur public, il serait trop long de les envisager tous dans un exposé de cette nature. Nous avons déjà rappelé que les gains du secteur public ont tendance à se répercuter dans le secteur privé. La question majeure est de savoir si le secteur privé pourra, sur le plan de la concurrence locale et internationale, suivre ce mouvement, et jusqu'à quel point. Si oui, tant mieux; si non, on sait qui en fera les frais. Sous d'autres aspects, comme celui de la sécurité d'emploi, il est à craindre que les deux régimes ne fassent que creuser davantage le fossé entre les travailleurs des deux secteurs. Il faut aussi envisager les conséquences sur d'autres salariés de certaines mesures qui se développent dans le secteur public. Pour remplacer les salariés réguliers en congé de maternité, d'éducation, d'activités syndicales ou autres, il faudra d'autres salariés, occasion-

49 Loi sur les normes du travail. (Projet de loi $n^{\circ} 126$, sanctionné le 22 juin 1979). Lois $d u$ Québec, 1979, ch. 45. Loi sur la santé et sur la sécurité du travail. (Projet de loi $\mathrm{n}^{\circ} 17$, sanctionné le 21 décembre 1979). Lois du Québec, 1979, ch. 63). Charte des droits et libertés de la personne. (Projet de loi n ${ }^{\circ}$ 50, sanctionné le 27 juin 1975), Lois du Québec, 1975, ch. 6. Charte de la langue française. (Projet de loi ${ }^{\circ} 101$, sanctionné le 26 août 1977), Lois du Québec, 1977, ch. 5. Loi de la qualité de l'environnement, Lois du Québec, 1972, ch. 49.

so CÔTÉ, André C., "Les recours en exécution: accessibilité et réalisme?», dans La détermination des conditions minimales de travail par l'État, compte rendu du $35^{\mathrm{e}}$ congrès des relations industrielles de l'Université Laval, Québec, Les Presses de l'Université Laval, 1980, 229 p., pp. 81-109. 
nels, temporaires ou surnuméraires, qui ne sauraient avoir, par définition, les mêmes avantages, et qui n'auront sûrement pas les mêmes garanties d'emploi que les salariés réguliers. Faudra-t-il un nouveau mouvement pour leur assurer, à eux comme aux autres, des conditions de travail raisonnables? Il peut paraître souhaitable, à prime abord, que le syndicat sur place se charge de la défense de tous les salariés, les réguliers et leurs aides; dans le concret, on risque fort de retrouver des querelles à l'intérieur même du syndicat: les salariés réguliers et les autres ont souvent des intérêts divergents, sinon carrément contradictoires les uns par rapport aux autres. Rien n'étant gratuit, si ce n'est l'air, le soleil et l'eau de nos rivières - et encore! - tout avantage que la négociation collective assure à un salarié impose une contrainte correspondante à quelqu'un d'autre, le plus souvent un salarié lui aussi. L'observation ne signifie pas qu'il faille renoncer à défendre ses droits et même ses intérêts, mais simplement qu'il faut aussi mesurer les implications de tout avantage garanti à tel groupe de personnes.

\section{Conclusions}

En résumé, les coordonnées de base du processus de la négociation n'ont guère changé depuis 1970. Il n'y a pas eu de modifications vraiment majeures à la situation au cours de la décennie: toutes les institutions de base avaient été mises en place dans les années 1960. D'un autre côté, un clivage profond s'est effectué entre la négociation dans le secteur public et celle du secteur privé, à l'avantage du public sur le privé. Cet avantage pourrait tenir au niveau du syndicalisme, quasi complet dans le public, encore très faible dans le privé; il ne faudrait pas oublier cependant que le syndicalisme dans le secteur public s'est fait par législation, réglementation et convention, beaucoup plus que par la volonté et les efforts des travailleurs euxmêmes comme dans le secteur privé. Le secteur de la construction ressemble, sur ce point, au secteur public.

L'observation rejoint le débat sur la négociation sectorielle. Commencée à la fin des années 1960, la discussion d'un tel projet s'est poursuivie avec intensité au début de $1970^{51}$. Elle refait surface de temps à autre ${ }^{52}$. Des

51 SAUVÉ, Robert, "La négociation sectorielle», Relations industrielles, 26, 1, janvier 1971, pp. 3-23. Robert SAUVÉ, "Vers la négociation sectorielle», Québec/Travail, 6, 5, novembre-décembre 1970, pp. 16-19. Marcel PÉPIN, «Propositions générales concernant la négociation sectorielle", Rapport sur la négociation sectorielle. $44^{\mathrm{e}}$ congrès, C.S.N., du 6 au 12 décembre 1970. Charles PERRAULT, «Le bilan de la négociation sectorielle au Québec», Bulletin des Conseillers en relations industrielles, janvier 1974.

52 ROBACK, Léo, La syndicalisation sectorielle. Pour une solution à l'organisation des non syndiqués. Montréal, Institut de recherche sur le travail (I.R.A.T.), Bulletin $\mathrm{n}^{\circ} 10$, février 1977, 48 p. Bernard BRODY et Gilles CARDIN, «La négociation sectorielle: la problématique», dans La gestion des relations du travail au Québec, publié sous la direction de Noël Mallette, Montréal, McGraw-Hill, pp. 497-511. 
rumeurs voudraient qu'on y recoure bientôt pour régler les problèmes de l'industrie du vêtement. On devrait analyser de plus près l'expérience de la construction avant de s'embarquer dans une autre aventure semblable. L'effet assuré, c'est la syndicalisation obligatoire. Les autres résultats sont aléatoires.

La syndicalisation et la négociation sectorielles peuvent s'avérer illusoires. Portée à un tel niveau d'envergure, que reste-t-il de la négociation collective au sens traditionnel du mot, c'est-à-dire de deux agents économiques qui cherchent à déterminer des conditions de travail qui soient les meilleures et demeurent compatibles avec le prix du produit sur le marché. On a dit que les pressions politiques se comparaient, dans le secteur public, aux pressions économiques du secteur privé. Même sous cet angle, les pressions peuventelles modifier la masse salariale de l'État? Après qu'un gouvernement a établi son budget et sa politique salariale, que celle-ci s'appelle formule Parizeau ou autre chose, un groupement syndical a-t-il jamais réussi à changer substantiellement la proposition de l'État? Son pouvoir est considérable, puisque ses membres ont à toutes fins pratiques la sécurité d'emploi et qu'ils assurent souvent un service essentiel, dont les prestations ne peuvent se suspendre longtemps. D'un autre côté, l'État responsable du bien public peut et doit venir appuyer l'État employeur, sous peine de risquer de graves bouleversements dans la société. Il l'a fait, et il continuera de le faire, c'est inévitable.

Mais de nouveau, peut-on vraiment appeler de tels affrontements politiques de la négociation collective? On a, d'une part, un mouvernent syndical dont la force de frappe est considérable, par suite des services stratégiques qu'assurent ses membres et de la garantie d'emploi qui leur est déjà donnée, d'autre part, un État qui ne peut tolérer d'arrêts de travail prolongés dans certains secteurs, parce que la vie de l'ensemble de la population en deviendrait trop difficile, et que celle-ci ne saurait finalement le supporter.

Le problème des relations du travail rejoint ainsi les options fondamentales de la société. Depuis plusieurs décennies, la société, par sa législation sur les relations du travail, a favorisé le mouvement syndical plus qu'elle ne lui a imposé de contraintes; la décennie de 1970 ne fait pas exception. Pour une raison ou pour une autre, le mouvement syndical s'est radicalisé. Les gains réalisés tant par la loi que par les conventions collectives ont éveillé des appétits de plus en plus grands; les profits de certaines entreprises ont aggravé ce sentiment encore davantage. Par contre, la multiplication des arrêts de travail dans le secteur public a exaspéré la population. Il faudrait être aveugle et sourd pour ne pas voir et entendre l'exaspération impuissante de cette population; sa colère vise indifféremment les administrateurs et les syndiqués. Si elle ne réagit pas et endure en silence, c'est qu'elle est sans voix 
et sans mains. Tout ce qu'on observe, c'est un certain mouvement de l'opinion publique vers la droite. Ce mouvement produira-t-il des résultats? Personne ne le sait vraiment. En tout cas, on ne peut pas s'attendre à de profonds changements dans un avenir prochain. Mais si le mouvement acquiert un jour un certain pouvoir, une des premières institutions qu'il remettra en cause ce sera la libre négociation collective, tout spécialement dans le secteur public.

Autour des années 1970, on pouvait lire des articles qui s'interrogeaient si la convention collective avait encore des chances de survivre, si ce n'était pas un mode bientôt désuet de déterminer les conditions de travail ${ }^{53}$. En 1980 , on ne voit guère d'articles de cette nature, et pourtant la question est peut-être plus pertinente que jamais, surtout au Québec.

$\mathrm{Au}$ fond, c'est qu'il existe un hiatus profond entre les objectifs de la libre négociation collective et la façon dont elle se vit chez nous, surtout dans le secteur public. Une situation fausse entre des principes et une pratique appelle tôt ou tard des rajustements. La solution la plus avantageuse est toujours celle qui vient volontairement des intéressés. Mais dans le cas qui nous occupe, on ne peut guère entretenir d'espoir quant à ce genre de solution: l'opposition entre les parties semble s'aggraver plutôt que s'atténuer. Les risques d'éclatement ne sont pas encore assez clairement perçus pour amener chaque partie à faire les concessions requises, encore une fois surtout dans le secteur public. D'un autre côté, l'inaction des intéressés risque d'appeler éventuellement une intervention drastique, que personne ne souhaite vraiment.

\section{LES CONVENTIONS COLLECTIVES}

Il reste à évaluer le résultat des négociations collectives. Quelle a été l'évolution, au cours de la décennie de 1970, du contenu des conventions collectives? Avant de revoir les principales clauses une à une, il faut relever quelques observations générales.

53 FEINSINGER, Nathan, "Is the American Collective Bargaining System Obsolete?", Proceedings of the Seventeenth Annual Meeting of the Industrial Relations Research Association, Chicago, Ill., December 28-29, 1964. Madison, Wis., I.R.R.A., 1965, pp. 156-159. James D. HODGSON, "The Survival of Collective Bargaining", dans Collective Bargaining: Survival in the '70's? Proceedings of a Conference Sponsored by the Industrial Research Unit and the Labor Relations Council, Wharton School, University of Pennsylvania Press, c. 1972, pp. 3-9. "La négociation collective sur la sellette». Trois articles parus dans La Gazette du Travail, 71, 1, janvier 1971, pp. 14-25. Plus récemment "A Creaky System of Collective Bargaining", Business Week, June 30, 1980, pp. 82-83. 


\section{Analyse des conventions collectives}

La décennie de 1970 a connu un essor remarquable dans l'analyse des conventions collectives. Depuis la fin des années 1960, Travail Canada publie, chaque année, un relevé statistique des principales dispositions, clause par clause, des grandes conventions collectives selon les différentes industries $^{54}$. La population analysée jusqu'ici se limitait aux conventions visant 500 travailleurs et plus; on doit bientôt en étendre la portée. L'avantage majeur de cette série, c'est qu'elle permet de dégager une certaine évolution historique: les premières publications remontent à une quinzaine d'années; même si la grille d'analyse s'est modifiée assez considérablement au cours de la période, des comparaisons temporelles valables demeurent possibles.

À la suite des initiatives de Travail Canada, les provinces ont emboîté le pas au cours de la décennie. À l'heure actuelle, la plupart des provinces canadiennes ont un système informatisé d'analyse des conventions collectives $^{55}$. Même des organismes privés, syndicats et bureaux de recherche, se sont lancés dans cette voie ${ }^{56}$.

Au Québec, l'effort d'analyse des conventions collectives est relativement récent: il a commencé il y a deux ans, et la première publication a paru en juin 1980; elle portait sur environ 1,500 conventions collectives en vigueur au Québec en 197957. L'analyse faite au Québec vise l'ensemble de la population des conventions collectives; les résultats reflèteront donc le contenu des conventions qui régissent non seulement les grandes unités, mais même les plus petites. Mentionnons, en passant, que $60 \%$ des conventions

54 Travail Canada, Dispositions de grandes conventions collectives concernant les employés dans l'industrie manufacturière au Canada, 1969, 1974, 1979, Ottawa, ministère du Travail du Canada, Direction des données sur le travail, Division de la négociation collective. Dans les années intercalaires, on a publié, selon la même grille d'analyse, les données correspondantes pour d'autres industries. Pour la fonction publique fédérale, voir l'excellente série publiée par le Bureau de recherche sur les traitements: Analyse des conventions collectives dans la fonction publique fédérale.

55 Mentionnons les publications des provinces suivantes: Ontario Ministry of Labour, Bargaining Information Series. Chaque fascicule porte un titre différent selon la clause analysée. British Columbia Ministry of Labour, Negotiated Working Conditions. Nova Scotia Department of Labour, Negotiated Working Conditions. Pour le Québec, voir la note 57.

56 Les Métallurgistes unis d'Amérique et le Syndicat canadien de la Fonction publique ont institué leur propre système informatisé d'analyse des conventions collectives. Un bureau privé de Kingston a commencé, en 1980, la publication d'une série fort utile, basée sur les données de Travail Canada, mais qui déborde l'aspect statistique: Collective Agreement Series, I.R. Research Services, Kingston, Ontario.

57 Québec, Conditions de travail contenues dans les conventions collectives au Québec, 1979, Québec, ministère du Travail et de la Main-d'oeuvre, Centre de recherche et de statistiques sur le marché du travail, 1980. La publication compte sept cahiers, dont le premier vise l'ensemble des secteurs, et les autres chacun un secteur particulier. 
collectives en vigueur au Québec visent des groupes d'employés de 50 salariés ou moins; il était donc impérieux d'en connaître le contenu. Par contre, ces conventions collectives ne régissent que $12 \%$ de l'ensemble des salariés soumis à des conventions collectives ${ }^{58}$.

Pour faire des comparaisons et, à plus forte raison, pour dégager des tendances, on ne peut, à ce moment-ci, utiliser les résultats de l'analyse faite au Québec qu'avec précaution. La banque de données n'est pas complète on n'a pas encore codifié l'ensemble des quelque 6,000 conventions différentes présentement en vigueur - et la sélection des conventions déjà codifiées s'est faite selon des impératifs administratifs plutôt que selon un échantillon scientifique. La publication des données relatives à 1980 devrait paraître sous peu; elle présentera une distortion majeure. Fondée sur 3,000 conventions en vigueur en 1980 , elle inclura les conventions du secteur public et parapublic. La différence qui existe entre les conventions du secteur public et celles du secteur privé, tant par leur contenu que par l'étendue de leur application, va rendre toute comparaison entre les données fournies dans la publication de 1979 et dans celle de 1980 extrêmement périlleuse. En particulier, on ne saurait conclure à la présence de quelque tendance que ce soit par rapport à l'étendue d'application d'un type de clauses à partir de cette seule comparaison.

Pour toutes ces raisons, l'analyse qui suit présentera des impressions générales plutôt que des conclusions définitives, même si toutes les données disponibles ont été scrutées avec la plus grande rigueur possible.

\section{La forme des conventions collectives}

On peut distinguer le fond et la forme des conventions collectives, soit leur contenu et leurs autres caractéristiques. On peut également distinguer la forme interne et la forme externe des conventions.

Par forme interne des conventions, j'entends les caractéristiques générales des conventions elles-mêmes: leur longueur, le caractère juridique de leur formulation, et l'interprétation légaliste qu'on en a donnée. Ces trois aspects se sont accentués au cours de la dernière décennie: les conventions se sont allongées, leur formulation est devenue de plus en plus technique et juridique, et l'interprétation qu'on en a faite semble de plus en plus légaliste. Rien ne laisse prévoir un changement d'orientation à ce sujet, même si la tendance présente entraîne beaucoup de difficultés et que plusieurs souhaiteraient une approche différente aux problèmes de relations du travail. 
Quant à la forme externe des conventions, j'entends par là principalement l'étendue de leur application. Je songe en particulier à l'opposition qui existe entre la convention dont l'application est limitée à un établissement et, à l'autre extrémité, la convention dite sectorielle. Les années 1970 ont vu la mise en place de conventions sectorielles dans le secteur public et parapublic, ainsi que dans l'industrie de la construction. La portée et l'impact de ces conventions collectives sont sans commune mesure avec les conventions d'entreprise ou d'établissement. Dans les analyses statistiques, on reconnaît leur influence dans le nombre considérable de salariés qu'elles impliquent, et dans la différence que ce nombre entraîne entre le pourcentage des conventions collectives et le pourcentage des salariés dans les divers tableaux de distribution de fréquence.

\section{Le contenu des conventions collectives}

Nous considérerons maintenant les principales clauses de conventions collectives, pour en dégager l'évolution récente ${ }^{59}$. Nous regrouperons les clauses de la façon suivante: clauses monétaires et avantages sociaux, mouvements de personnel, sécurité au travail et sécurité d'emploi, enfin les clauses dites conventionnelles, qui visent soit la convention collective elle-même soit les parties signataires.

\section{Clauses monétaires}

Par rapport aux salaires, si on part d'un peu plus loin en arrière, pour agrandir la perspective, on peut noter que de 1960 à 1975, les salaires, et surtout les salaires négociés, ont augmenté beaucoup plus vite que le coût de la vie, plus précisément que l'indice des prix à la consommation ${ }^{60}$. Depuis l'introduction des mesures anti-inflationnistes, en 1975, et peut-être davantage depuis leur suppression, le niveau des salaires et l'indice des prix à la consommation ne suivent plus un mouvement uniforme. En simplifiant beaucoup, on peut dire que les deux ont augmenté plus ou moins également: le niveau des salaires réels s'est à peu près maintenu; plus récemment, certaines conventions n'ont même pas réussi à suivre l'augmentation des prix.

59 Les pages qui suivent reposent principalement sur les publications mentionnées dans les notes 54 et 57, ainsi que sur les données mécanographiques du ministère du Travail et de la Main-d'oeuvre du Québec relatives aux conventions collectives en vigueur au Québec en 1980. Pour quelques clauses, certains ouvrages traitant de points particuliers ont été utilisés; ils sont mentionnés dans les notes suivantes.

60 HÉBERT, Gérard, "L'impact de l'inflation sur la négociation collective», Inflation, indexation et conflits sociaux, $30^{\mathrm{e}}$ congrès des relations industrielles, Université Laval, 1975, pp. 49-96. 
Avec la flambée de l'inflation, au milieu de la décennie, les clauses d'indexation, surtout dans les grandes conventions collectives, ont augmenté en nombre et en proportion de façon considérable ${ }^{61}$. En même temps, la durée des conventions diminuait. Avec la persistance de l'inflation, on s'y est presque habitué. Les conventions se sont rallongées quelque peu; la majorité d'entre elles ont présentement une durée de deux ou trois ans. Le nombre des clauses d'indexation n'augmente plus; il semble même diminuer un peu. Phénomène plus récent encore, les clauses d'indexation semblent se diversifier.

Toutes les primes augmentent, non pas de façon spectaculaire, mais elles s'améliorent constamment. Par exemple, un plus grand nombre de clauses prévoient le paiement du salaire à taux double, après un nombre d'heures supplémentaires moins élevé à taux et demi. Les primes de postes augmentent, les primes d'heures brisées, de rappel, etc. Notons la présence de certaines dispositions un peu nouvelles, qui deviennent suffisamment importantes pour retenir l'attention des codificateurs, comme la garantie de salaires en cas de bris d'équipement. Mentionnons également une prime nouvelle, d'une autre nature, une prime d'assiduité, différente du paiement des congés de maladie non utilisés. Selon cette clause, le travailleur qui ne s'est pas absenté du travail et à qui on ne peut reprocher aucun retard jouit d'un bonus particulier, qui lui est remis avec sa paie de vacances.

Dans les heures de travail, on observe une tendance à trois dimensions. Pour les ouvriers à la production, la semaine normale demeure stable à 40 heures dans la plupart des cas. Chez les cols blancs, on observe une légère diminution, mais la grande majorité demeure autour de 35 heures. Sous un autre aspect, on assiste à une campagne de publicité en faveur de la diminution de la semaine normale de travail. Tous les arguments traditionnels y sont repris, en particulier la répartition du travail disponible et la diminution possible du chômage. Mais la semaine normale de travail ne change guère. L'évolution se fait plutôt par rapport à l'ensemble du temps payé et non travaillé (vacances, congés de toutes sortes) que sur le point précis de la semaine de travail. Sur celui-ci, il faut mentionner les diverses expériences d'horaires flexibles, ou de semaine comprimée, dont le nombre s'accroît,

61 ASCAH, Louis, "La diversité des clauses d'indemnité de vie chère dans les conventions collectives", Relations industrielles, 35, 1, 1980, pp. 121-127. Jean-Michel COUSINEAU et Robert LACROIX, L'indexation des salaires, Monographie $\mathrm{n}^{\circ} 10$, Université de Montréal, École de relations industrielles, c. 1981, 119 p. David A. WILTON, An Analysis of Canadian Wage Contracts with Cost-of-Living Allowance Clauses, Discussion Paper no. 165, Ottawa, Conseil économique du Canada, mars 1980, $40 \mathrm{p}$. 
sans qu'on puisse parler d'une tendance prononcée. Deux mots résument l'évolution quant à la durée hebdomadaire du travail: stabilité relative et expériences de réaménagement ${ }^{62}$.

Comme corollaire à la question des heures, on peut relever la réglementation du travail effectué en temps supplémentaire. Les conventions collectives ont tendance à préciser davantage comment les heures de travail supplémentaire seront réparties ou offertes aux employés, et surtout à garantir aux travailleurs le droit de refuser d'effectuer du travail en heures supplémentaires.

\section{Avantages sociaux}

Un domaine où l'évolution a été marquée et continuera sans doute de l'être, c'est celui des avantages sociaux, tout particulièrement l'augmentation du temps rémunéré mais non travaillé.

Les vacances annuelles se sont prolongées considérablement. Par exemple, la disposition prévoyant quatre semaines de vacances annuelles après dix années de service ne se retrouvait, il y a dix ans, que dans $3 \%$ des grandes conventions collectives; aujourd'hui on la rencontre dans $25 \%$ des cas. La durée des années de service requise pour avoir droit à un certain nombre de semaines de vacances s'est également raccourcie: plusieurs conventions, à l'exemple du secteur public, accordent maintenant quatre semaines de vacances après une seule année de service. On trouve des cas de cinq et six semaines de vacances, pour les ouvriers ayant travaillé quinze ou vingt ans pour le même employeur.

On se rapproche du congé sabbatique, que les Métallurgistes unis d'Amérique avaient essayé d'implanter, mais sans grand succès, il y a une quinzaine d'années. Le niveau de qualification de plus en plus élevé que réclament les emplois nouveaux pourrait bien favoriser la multiplication des clauses de cette nature. La disposition fait partie des différentes formes que prend, à l'heure actuelle, la réduction globale de la durée du travail, par opposition à sa durée journalière ou hebdomadaire.

Le nombre de jours fériés semble relativement stable, de 10 à 12 par année. Les conventions visant un plus grand nombre d'employés tendent à assurer une journée ou deux de plus que les petites conventions. L'habitude

62 Congrès des relations industrielles, L'aménagement des temps de travail, $29^{\mathrm{e}}$ congrès des relations industrielles de l'Université Laval, Québec, Les Presses de l'Université Laval, 1974, 337 p. Congrès des relations industrielles, Le temps au travail: un facteur compressible?, $36^{\mathrm{e}}$ congrès des relations industrielles de l'Université Laval, Québec, Les Presses de l'Université Laval, 1981. 
syndicale de demander une journée supplémentaire, à chaque ronde de négociation, ainsi que la coutume patronale d'accéder à une telle demande en échange d'une concession dans un domaine jugé plus important, assurent une augmentation lente mais constante du nombre de congés fériés.

Les périodes de repas et les périodes de repos sont plus souvent mentionnées qu'auparavant, mais leur durée demeure relativement stable. Pour les périodes de repos, la règle la plus générale est de deux périodes de quinze minutes dans chaque demi-journée de travail.

Le domaine qui a connu le développement le plus spectaculaire au cours de la décennie semble bien être celui des congés familiaux et sociaux. Les grandes innovations, comme le congé prolongé de maternité, sont apparues dans le domaine public d'abord. Plusieurs éléments sont en train de passer, mais à un rythme beaucoup moins rapide, dans les conventions collectives du secteur privé. Le transfert d'un secteur à l'autre se continuera probablement au cours des prochaines années, mais il n'est pas sûr que le nombre et l'importance de ces congés continuent d'augmenter: les coûts qu'ils représentent n'avaient pas été, semble-t-il, correctement estimés, et toutes les répercussions de ces clauses ne sont pas encore connues et évaluées.

Si le nombre des congés de maladie accordés par les conventions collectives a augmenté, on a observé dans ce domaine le rappel de certains avantages autrefois assez répandus. En effet, les banques de congés de maladie ont tendance à disparaître. Les congés qui n'ont pas été utilisés au cours de l'année sont perdus ou monnayés, en tout ou en partie. La protection qu'offraient les banques de congés de maladie contre une maladie de longue durée est remplacée par différents programmes d'assurance-salaire.

Les clauses d'avantages sociaux relatives aux plans d'assurances collectives et de pensions se sont légèrement accrues, et leurs dispositions modérément améliorées. Mentionnons, tout particulièrement, que le nombre de conventions collectives prévoyant une assurance couvrant les soins dentaires augmente.

D'autres domaines, que l'on peut rattacher aux avantages sociaux, manifestent également une faible croissance, sinon une relative stabilité, par exemple les indemnités de licenciement. Pour souligner la différence entre les grandes et les petites conventions collectives, notons que 50\% des grandes conventions contiennent une disposition relative aux indemnités de licenciement, alors que seulement $20 \%$ de l'ensemble des conventions collectives en vigueur au Québec, et analysées en 1979 et 1980, contiennent une telle clause. 


\section{Mouvements de personnel}

L'importance de l'ancienneté dans les clauses de mises à pied et de promotions a tendance à augmenter. La tendance est très nette dans les cas de mises à pied; même plus faible, elle existe relativement à l'avancement et aux promotions. La période pendant laquelle les employés conservent leur ancienneté, en cas de mises à pied ou de maladie, tend également à s'allonger; jusqu'ici, l'employé conservait ses droits le plus souvent pendant douze mois ou moins, présentement c'est plutôt au-delà de douze mois que cette conservation se prolonge.

Sur un point connexe, mentionnons ce qui paraît être, du moins à première vue, un gain pour la partie patronale: la durée de la période de probation semble s'allonger quelque peu. Le nombre de cas prévoyant 30 jours ou moins diminue, alors que les cas où la durée se situe de 30 à 60 jours augmentent. La modification ne reflète pas nécessairement et uniquement une victoire patronale: elle peut découler d'une transformation des emplois, c'est-à-dire d'un accroissement relatif des fonctions qui requièrent une plus haute compétence et, en conséquence, une période de probation plus longue.

\section{Sécurité au travail et sécurité d'emploi}

Sans doute à cause de la Loi 17 sur la santé et la sécurité au travail, les clauses relatives à ce sujet ont augmenté considérablement au cours des dernières années. Les parties veulent préciser, pour leurs besoins respectifs, les dispositions générales que la loi contient. Ces précisions portent le plus souvent sur des questions d'équipement de sécurité, et sur la formation et le fonctionnement des comités conjoints imposés par la loi. Certaines conventions contiennent également des dispositions sur d'autres aspects de la santé et de la sécurité au travail. Ces clauses servent en quelque sorte de compléments à la loi.

C'est en matière de sécurité d'emploi qu'on note les divergences les plus grandes entre les conventions du secteur public et celles du secteur privé. En effet, seules les institutions du secteur public sont assurées de survivre et peuvent ainsi garantir à leurs employés une sécurité totale d'emploi. C'est peut-être l'aspect qui marquera le plus profondément la différence des conditions de travail entre les deux secteurs dans les années à venir. Bien que les entreprises privées ne soient jamais assurées de leur survie, leurs employés cherchent quand même à protéger leurs emplois de la façon la plus efficace possible. Cette protection vise principalement deux menaces différentes, les changements technologiques et les sous-contrats. 
En matière de changements technologiques, tout comme dans le domaine de la santé et de la sécurité au travail, les lois imposent déjà diverses obligations aux employeurs, en particulier celle de donner un préavis aux représentants de leurs employés en cas de fermeture ou de licenciement collectif important ${ }^{63}$. Les parties doivent alors établir un comité qui essaiera de minimiser les effets négatifs de la décision prise. Les conventions collectives précisent habituellement la durée du préavis, souvent dans l'intention de l'allonger: la règle la plus fréquente est celle de 3 à 6 mois.

Le problème des sous-contrats est différent, et il affecte certaines catégories d'employés de façon plus directe que d'autres. C'est ainsi que le secteur municipal est un de ceux où les clauses de sous-contrats se sont accrues davantage au cours des dernières années. Mais les clauses de sous-contrats se retrouvent dans toutes les industries. Dans l'industrie manufacturière, le nombre et la proportion des conventions collectives contenant une telle clause est passé de $33 \%$ à $50 \%$, au cours des dix dernières années. Règle générale, la clause vise à limiter ou à réglementer l'octroi des sous-contrats: la condition la plus fréquente est que ceux-ci ne doivent pas causer de mises à pied dans l'unité de négociation visée ${ }^{64}$.

\section{Clauses conventionnelles}

Nous avons déjà noté plus haut que la poussée inflationniste avait réduit quelque peu la durée moyenne des conventions collectives. Parmi les grandes conventions collectives, la grande majorité ont une durée de deux ou trois ans, avec une légère prédominance des conventions de deux ans. Plus la convention est longue, plus on a de chance d'y trouver une clause d'indexation. Une autre façon de se prémunir contre les aléas de l'inflation, tout en signant une convention collective de longue durée, c'est d'y introduire une clause de réouverture pour les salaires. Cette formule est cepen-

63 Loi sur la formation et la qualification professionnelles de la main-d'oeuvre. (Projet de loi $\mathrm{n}^{\circ} 49$, sanctionné le 13 juin 1969), Lois du Québec, 1969, ch. 51, art. 45 (Lois refondues $d u$ Québec, ch. F-5, art. 45). L'article 45 a été modifié par la Loi modifiant la Loi sur les normes du travail et la Loi sur la formation et la qualification professionnelles de la maind'oeuvre. (Projet de loi n ${ }^{\circ}$ 91, sanctionné le 10 avril 1980), Lois du Québec, 1980, ch. 5 art. 12 et 13. Code canadien du travail, Partie V, Statuts refondus du Canada, ch. L-1, art. 149-153.

64 Même si la controverse sur la sous-traitance s'est intensifiée au cours des dernières années, aucune étude d'importance sur le sujet n'a été effectuée. On se réfère encore à l'ouvrage de F. John L. YOUNG, Contracting Out of Work. Canadian and U.S.A. Industrial Relations Experience, Research Series No. 1, Kingston, Queen's University, Industrial Relations Centre, $1964,150 \mathrm{p}$. Parmi les nombreux mémoires présentés à divers niveaux de gouvernement sur le sujet, mentionnons celui du Conseil pour l'expansion de l'industrie de la construction: Report and Recommendations of the Task Force on Contracting Out, Ottawa, Construction Industry Development Council, 1980, 21 p. 
dant moins fréquente. Avec la persistance de l'inflation, on ne peut s'attendre à des changements majeurs dans la durée des conventions collectives, à moins que l'introduction de contrôles économiques ou un changement radical de contexte n'introduise un élément supplémentaire d'incertitude qui amène quelques parties à raccourcir la durée de leur convention.

En matière de sécurité syndicale, les clauses qui imposent l'adhésion obligatoire ont augmenté au cours des dix dernières années, passant de $66 \%$ à $75 \%$. C'est dans l'atelier syndical que se retrouve principalement cette augmentation, les autres formules étant relativement stables. Il serait étonnant de voir des changements spectaculaires sur ce point. En général, les entreprises et les institutions qui ont refusé jusqu'ici de rendre l'adhésion syndicale obligatoire l'ont fait pour une raison de principe; elles ne seront guère disposées à modifier leur position sur le sujet. Par contre, celles qui l'acceptent sont généralement prêtes à accorder une forme ou l'autre d'atelier syndical. La retenue syndicale, rendue obligatoire par une disposition du Projet de loi $45^{65}$, fait toujours l'objet de précisions dans les conventions collectives.

Un aspect particulier de la sécurité syndicale a reçu une attention particulière, surtout dans le secteur public, au cours des dernières années. Dans l'ensemble des conventions collectives, les libérations syndicales augmentent légèrement; mais cette augmentation est substantielle, à tous égards, dans le secteur public et parapublic.

En matière de règlement des griefs, plus précisément par rapport à l'arbitrage, on peut noter un certain accroissement du nombre et de la proportion des conventions qui prévoient la nomination d'un arbitre unique plutôt que d'un conseil d'arbitrage. On trouve également, depuis quelque temps, une mention légèrement plus fréquente de la possibilité de recourir à l'arbitrage accéléré. Rien ne permet de conclure, cependant, qu'on y recourt effectivement beaucoup plus.

On rattache habituellement aux clauses conventionnelles la création de comités mixtes ou conjoints. On a pu observer, à ce sujet, un accroissement important des clauses de cette nature. Notons ici, encore une fois, la différence entre les grandes conventions collectives et la totalité des conventions en vigueur au Québec: les proportions sont, respectivement, de $75 \%$ et de $30 \%$.

65 Loi modifiant le Code du travail et la Loi du ministère du Travail et de la Maind'oeuvre. (Projet de loi n ${ }^{\circ} 45$, sanctionné le 22 décembre 1977), Lois du Québec, 1977, ch. 41, art. 28 modifiant l'art. 38 du Code et ajoutant les articles 38 a et $38 \mathrm{f}$. (Lois refondues $d u$ Québec, 1977, ch. C-27, art. 47 à 47.6). 
Depuis l'adoption de la Loi 17 , ces comités visent le plus souvent des questions de santé et de sécurité au travail. On trouve quelques cas de comités mixtes de production. Notons, en passant, que cette expression, moins appréciée de nos jours, est souvent remplacée par d'autres mots; la nouvelle expression aux États-Unis est celle de "Quality Circles" 66 . Enfin, il faut signaler les nombreux comités de relations de travail, connus sous divers noms, avec ou sans objet précis. Qu'on les appelle comités de relations humaines, comités de relations patronales-syndicales, comités de relations professionnelles, ou autrement, ils ont toujours pour objectif, finalement, d'améliorer la communication et les rapports entre les deux parties. Certains préfèrent leur donner un objet précis et limité, d'autres aiment mieux laisser aux participants le soin de déterminer la forme et le contenu de leurs rencontres. Sans atteindre des proportions extraordinaires, le nombre de ces comités augmente d'une façon assez substantielle pour laisser entrevoir une évolution intéressante. Elle manifeste la présence d'un esprit qui pourrait affecter profondément l'évolution des relations du travail dans les années à venir. Nous y reviendrons dans la prochaine et dernière section de cet exposé, qui traite des paramètres des orientations futures.

\section{Paramètres des orientations futures}

Pour estimer si les orientations mentionnées jusqu'ici se poursuivront ou si elles risquent d'être modifiées profondément, il convient d'examiner, en terminant, le contexte dans lequel évolueront les négociations au cours des prochaines années.

Le facteur le plus important qui pourrait modifier le contenu des négociations dans les années à venir, c'est l'évolution du contexte économique. La persistance des taux d'inflation autour de $10 \%$ à $12 \%$ et l'augmentation constante des taux d'intérêts posent la question d'un contrôle possible des prix, des salaires, des profits et des taux d'intérêts. Il ne s'agira pas nécessairement de contrôles tels que nous les avons connus de 1975 à 1978; mais plusieurs considèrent que, politiquement parlant, les gouvernements devront intervenir. Les options possibles se situent toujours entre deux extrêmes, soit le gel des prix et des salaires, avec une détermination d'autorité des taux d'intérêts et des taux d'échange, soit, à l'autre extrême, la simple incitation. On peut prévoir qu'une intervention se situerait entre ces deux extrêmes. Quelle que soit sa nature, elle imposera, de toute évidence, des contraintes à la négociation des conventions collectives. Selon les aspects qui seront réglementés, l'intérêt se portera sur d'autres groupes de clauses.

Un second facteur qui pourra influencer profondément l'évolution des conventions collectives, c'est la transformation du mouvement syndical. Au 
cours de la décennie de 1970 , il s'est produit un fractionnement du mouvement syndical comme on n'en avait jamais vu auparavant: des centrales nouvelles sont apparues, et la proportion des syndicats indépendants s'est accrue de façon extraordinaire. Les syndicats non affiliés représentent, depuis quelques années, près de $20 \%$ de l'ensemble des syndiqués du Québec, et la majorité des demandes d'accréditation, à l'heure actuelle, viennent de groupes non affiliés. Les problèmes actuels dans le secteur de la construction, tant au Québec que dans l'ensemble du Canada, vont dans le même sens, celui d'un émiettement du mouvement syndical. Le phénomène ne peut pas ne pas influencer la négociation et le contenu des conventions.

D'autres caractéristiques du mouvement syndical exerceront également leur influence. Mentionnons, par exemple, l'opposition qui s'exprime maintenant au grand jour entre les permanents et les élus, le recours de plus en plus fréquent à des procédures judiciaires, même entre syndicats, la politisation de plus en plus grande de certains syndicats, et vraisemblablement, une offensive nouvelle pour obtenir de l'État une syndicalisation sectorielle obligatoire. Tous ces aspects se répercuteront évidemment à la table de négociation.

Le secteur public continuera d'influencer profondément le secteur privé, comme il l'a fait au cours des années 1970. D'un autre côté, il faut s'attendre à des modifications importantes dans les structures et les comportements du secteur public au cours des prochaines années. L'amélioration extraordinaire des conditions de travail qui a été obtenue au cours des trois dernières rondes de négociations, ainsi que les grèves majeures qui les ont accompagnées, ne sauraient continuer longtemps sans modifications. Deux voies semblent possibles. Ou bien nous assisterons à un ralentissement du phénomène, en ce sens que le cadre légal sera légèrement modifié, l'amélioration des conditions de travail sera plus modeste d'une ronde à l'autre, et les groupes syndiqués manifesteront plus de prudence dans le recours à des grèves qui affecteraient une partie considérable de la population. Ou bien l'on tentera de continuer le mouvement commencé au cours de la dernière décennie, et alors l'État se verra dans l'obligation d'adopter des solutions radicales. Diverses mesures ont été proposées; mentionnons la suivante, qui a le mérite de suggérer une formule claire. Les clauses monétaires seraient retirées du domaine de la négociation pour en remettre la détermination globale au Parlement ou à l'Assemblée nationale; quant aux clauses non monétaires, elles seraient négociées au niveau qui leur revient logiquement, c'està-dire au niveau local ou régional. Quelle que soit la formule qu'on adopte, les relations du travail dans le secteur public semblent destinées à subir des modifications profondes dans un avenir rapproché. De telles modifications se répercuteront directement dans les conventions collectives du secteur public, et par ricochet, dans les conventions du secteur privé. 
La législation normative qui s'est développée au cours des années 1970, surtout dans la deuxième partie de la décennie, continuera d'influencer considérablement les conventions collectives ${ }^{67}$. Nous avons déjà noté l'impact de la Loi sur la santé et la sécurité du travail. La Loi sur les normes, ainsi que les lois sur la non-discrimination, l'environnement et l'usage obligatoire de la langue française continueront d'exercer leur poids sur la négociation. Il ne faut pas croire, cependant, que toutes ces législations normatives amèneront les parties à retirer certaines clauses de la convention pour s'en remettre à la loi; bien au contraire, toute cette réglementation incitera plutôt les intéressés à en préciser l'application dans des clauses nouvelles, appropriées au contexte de chacun. Quand les gouvernements ont introduit le régime de pensions du Canada et le régime de rentes du Québec, certains ont cru que les plans de pension privée disparaîtraient des conventions collectives; c'est le contraire qui s'est produit: on a négocié des régimes particuliers dont le but était de compléter les régimes publics. On peut prévoir que la nouvelle législation normative aura des effets semblables, compte tenu de la nature propre de chaque cas.

Le cinquième et dernier paramètre que je voudrais mentionner est à la fois plus subtil et plus déterminant que les précédents, sauf peut-être l'environnement économique. Je veux parler du contexte des relations de travail et de l'approche fondamentale que la majorité des parties en cause adopteront. Il me semble que nous assistons à une évolution significative, même si elle n'est pas généralisée, de l'approche de base en relations du travail. Alors que le modèle nord-américain des relations de travail demeure fondé sur l'opposition et l'affrontement ("adversary system"), une autre approche se développe parallèlement, et semble gagner tranquillement de l'importance ${ }^{68}$. Cette autre approche prend différents noms. On parle de qualité de vie au travail (Q.V.T.); mais la réalité que vise ce mouvement dépasse considérablement ce que les mots laissent deviner. On parle également de relations humaines, de médiation préventive, de négociation continue. Nos

66 Business Week, May 11, 1981, p. 85.

67 Voir plus haut la note 49.

68 CONNAGHAN, Charles J., Association ou mariage de convenance?, Examen critique de l'état des relations professionnelles en Allemagne de l'Ouest, accompagné de suggestions en vue de l'amélioration des relations du travail au Canada à partir de l'expérience ouestallemande, Ottawa, Travail Canada, février 1977, 100 p. Hem C. JAIN, Worker Participation: Success and Problems, New York, Praeger, 1980, 358 p. Hem C. JAIN, "LabourManagement Commmittees and Workers' Participation", in Proceedings of the 17 th Annual Conference of the Canadian Industrial Relations Association, Montréal, 1980. Chris A. JECCHINIS, “Employees' Participation in Management. International Experiences and the Prospects for Canada", Relations industrielles, vol. 34, $\mathrm{n}^{\circ} 3,1979$, pp. 490-515. 
voisins anglo-canadiens ou américains parleront de "problem-solving approach", de "new industrial relations", de "labour relations by objectives" 69 .

Quels que soient les mots qu'on utilise, toutes ces expériences ont en commun qu'elles se fondent sur une plus grande communication, voire même sur une collaboration entre la direction et les employés, entre le patronat et le syndicat. L'expression "collaboration patronale-ouvrière» peut avoir perdu droit de cité, la réalité qu'elle recouvre n'en demeure pas moins une nécessité. Sans elle, il n'y a pas d'accroissement stable et prolongé de la productivité qui soit possible, pas plus que de rentabilité assurée pour l'entreprise, deux conditions fondamentales au mieux-être de toutes les parties en cause. Les discussions autour de la participation ouvrière vont dans le même sens. Le terme de co-gestion a une saveur trop juridique et trop contraignante. La réalité des relations de travail et de la production industrielle exige plus de souplesse.

La préoccupation de la nouvelle approche se manifeste, nous l'avons noté, dans la multiplication des comités mixtes de toutes sortes, que l'on trouve de plus en plus dans les conventions collectives. Dans la mesure où cette observation est valable et où le phénomène se poursuivra, la vie de l'entreprise dans son ensemble, direction et employés, patronat et syndicat, a chance de s'améliorer. De tous les paramètres susceptibles d'affecter les orientations futures des conventions collectives, c'est d'emblée le plus positif.

\section{Quebec Labour Relations in the 1970's}

Trends in collective bargaining have been ambiguous: certain events can be considered as major steps forward, which may in the long run jeopardize the future of free collective bargaining. Collective agreements have become longer, and more legalistic.

\section{Collective bargaining}

In terms of context, the following observations seem important. The proportion of unionized employees has remained substantially the same, but the proportion of unaffiliated unions has increased significantly. Inflation has been the number one phenomenon in the decade, with the wage controls in effect from 1975 to 1978 . The unemployment rate has remained fairly high in Quebec for the whole period, varying between 7 and $11 \%$.

69 KOCHAN, Thomas A., Collective Bargaining and Industrial Relations. From Theory to Policy and Practice, Homewood, Ill., Richard D. Irwin, Inc., 1980, 523 p., pp. 287, 430-435. "Special Report: The New Industrial Relations", Business Week, May 11, 1981, pp. 84-98. 
Besides a few major conflicts in the private sector, the whole decade has been dominated by the three bargaining rounds of the public and the parapublic sector. The proportion of man-days lost has hit a record-breaking $1.15 \%$ in 1976.

In the private sector, the A.I.B. controls have been the most influential element in the decade. These controls have caused the percentage of man-days lost to be, in 1977 and 1978, substantially lower than usual, and the average duration of the major collective negotiations relatively shorter. They have kept wage increases well below $10 \%$.

In labour law affecting private bargaining, the only major changes were made in Bill 45, enacted in 1977. The Bill has introduced voluntary conciliation, mandatory arbitration of the first collective agreement at the request of either party, the automatic application of the Rand formula upon certification, and the highly controversial provisions against strikebreakers. It is too early to evaluate the effects of these changes, especially those regarding strikebreaking.

Labour relations in the construction industry have been the object of a whole series of legislations. The introduction of a tight industry-wide bargaining scheme in the late '60's did not produce all the benefits that were expected. Government intervention was frequent and heavy to try and solve various difficulties arising out of the application of such legislation. Labour relations in the Quebec construction industry have become the most regulated in any industry anywhere in North America, certainly in the private sector.

In the public sector the basic rules had been laid down in the 1960's, like the right to strike in public and even essential services, but they received their final form only in the 1970's, especially through the laws and events of the three successive rounds of bargaining.

The 1972 round was the most highly centralized and the hardest-hitting of all. The Common Front of the various unions involved in the public sector was in full operation, the presidents of the three leading central bodies were jailed for recommending not to obey a special return-to-work law, social agitation was fairly high during May 1972, and the whole endeavour ended in the breaking up of the Common Front, the beginning of the expansion of unaffiliated associations, and the creation of a new central body.

The 1975-1976 and the 1978-1980 rounds were quite different. The Common Front was never as extended and as cohesive as in 1971-1972, and the strike activity was never as concentrated. On the whole, there may have been as many man-days lost, but they were much more scattered in time, sector and space. In all cases, a string of special return-to-work laws has been adopted, twelve in all over the decade, ten of which concerned the public sector.

The major characteristic of the various rounds is probably their political character. The objectives set down by the unions have a political connotation: they are openly aimed at raising the minimum working conditions of all employees, including those of the private sector. The process itself is highly political in the sense that the size and the impact of the whole exercise is bound to take up the colours of a major confrontation between the leading unions and the government. The 1972 slogan "We must break the system" is revealing of the very nature of the whole process. The effect is more economical than political, and the question mark is whether the private sector will be able to follow the leader with the major advantages granted to the public sector employees. 
Looking at the overall situation during the decade, the following general characteristics can be mentioned. The labour movement, while continuing to fulfill its bargaining agent responsibilities, has become much more radical in certain sectors. This has caused management, even public management, to take a harder line. The ritual of bargaining has remained much the same: negotiations are long and hard, and usually call for crisis bargaining before coming to an end. In many cases, confrontation is the name of the game. Work stoppages have become much more diversified than previously, legal or illegal, rotating or regular, wildcat or sympathy. Conversely, the religious respect given to a picket line, while remaining in most cases, is beginning to fade in certain instances. The adoption of various labour standards legislation has affected the scope of bargaining. Regarding the outcomes, the public sector agreements have influenced the private ones, at least in three major areas, the level of wage increases, the duration of annual holidays and the extent and conditions of maternity leave. The major difference concerns the security of employment: private companies can give no greater security to their employees than the one they enjoy themselves.

In conclusion, some events of the 1970's have raised serious questions concerning collective bargaining, at least in the public sector. Besides the political aspect that overshadow the determination of working conditions for these employees, the number and importance of work stoppages in public and essential services may eventually challenge the acceptability, if not the usefulness, of the whole process.

\section{Collective agreements}

The 1970's have seen important developments in collective agreement analysis. Labour Canada has continued and improved its survey and publications on the subject. Most of the Canadian provinces have put in place a computerized system of analysis of collective agreements. In Quebec, the whole population of collective agreements will eventually be analysed, thus making available a particularly important knowledge of the small collective agreements, the number of which is very high: $60 \%$ of all collective agreements in Quebec cover groups of 50 employees or less.

With regard to the format of collective agreements, their length and legalistic character have increased over the decade. Their coverage is extremely diversified: the vast majority of agreements regulate working conditions at the plant or the company level, while a few sector agreements, regarding public employees and the construction industry, govern working conditions for hundreds of thousands of employees.

Between 1960 and 1975, wages have increased more rapidly than the cost of living; since 1975, the picture is much more confused. Cost of living adjustment (C.O.L.A.) clauses have increased dramatically in the mid-1970's. The number of such clauses does not increase anymore; they tend to be more diversified. No dramatic change has occurred concerning premiums, working hours and overtime.

Fringe benefits have moved in a more significant way. The duration of annual holidays has increased, while the required number of years of service to enjoy longer vacations has decreased. A few paid holidays have been added to those already existing. Social holidays of all types (for funerals, wedding, births), which were almost non existent at the beginning of the decade, are now relatively frequent. The number of sick leave paid holidays has increased, but banks for unused sick leave holidays tend to disappear; they are replaced by some kind of wage insurance. Welfare plans and pension plans have been improved, while severance pay provisions are now somewhat more frequent than at the beginning of the decade. 
In promotions, transfers and lay-offs, seniority becomes more and more important. The period for retaining seniority rights, while on leave or on lay-off, also tends to increase. The length of the probation period has slightly increased, this being one of the few management gains over the period.

There has been an increase in the number of clauses pertaining to health and safety, probably due to the new legislation: collective agreements, either add to the minimum imposed by law, or make the provisions in the law more definite and specific for the parties. Employment security has become almost complete in the public sector, while it made only minor progress in the private sector; this progress was usually related to problems flowing from technological change. More collective agreements impose limitations on contracting out, especially in municipalities, but the content of the clause has remained substantially the same.

The inflation upsurge of the mid-1970's had a major effect on the duration of collective agreements: it increased the number and proportion of one-year agreements; but two and three year agreements have regained their previously favoured position, with a C.O.L.A. clause and/or a reopener to protect the employees against unexpected inflation. In union security, the union shop is becoming the predominant form in mandatory membership. The Rand formula now being obligatory by law, the collective agreements still contain the specifications on how to implement this obligation. Provisions for leave of absence on union affairs have increased substantially, in frequency and nature.

Concerning grievance procedure and arbitration, the only trend that can be noticed is a slightly increasing preference for the single arbitrator, as opposed to the arbitration council, and a somewhat more frequent recourse to expedited arbitration.

Finally, labour-management or joint committees have also increased in number and importance, especially in the larger agreements and concerning health and safety questions.

Whether these trends will continue depends a lot on the forthcoming context. The major factor will be the inflation situation, and the possible reintroduction of economic controls. The orientation of the labour movement will also be a major determinant of future labour relations. The weight of the public sector on the whole system will continue to be heavy, with a major question mark: will the public sector unions become more moderate, in the face of public reactions, or continue with always greater demands and greater confrontations? The possible development and consolidation of labour standards legislation will affect, one way or another, the content of future collective agreements.

The more important factor of all will likely be the fundamental approach to labour relations by the various parties involved. Even if the adversary system remains the basis of the general model, another approach implying a greater degree of cooperation is gaining importance. The various experiments in that direction carry different names: quality of working life, problem-solving approach, new industrial relations or labour relations by objectives. All these experiments have in common the belief in greater and more effective communication lines between labour and management. 